\title{
Electromagnetic deformable mirror development at TNO
}

\author{
Stefan Kuiper*a, Niek Doelman ${ }^{\mathrm{a}, \mathrm{b}}$, Evert Nieuwkoopa, Ton Overtoom ${ }^{\mathrm{a}}$, Tjeerd Russchenberg ${ }^{\mathrm{a}}$, \\ Martijn van Riel ${ }^{\mathrm{a}}$, Justin Wildschut ${ }^{\mathrm{a}}$, Max Baeten ${ }^{\mathrm{a}}$, Jet Human ${ }^{\mathrm{a}}$, Helma Spruit ${ }^{\mathrm{a}}$, Sanneke Brinkers ${ }^{\mathrm{a}}$, \\ Matthew Maniscalco ${ }^{\mathrm{a}}$. \\ ${ }^{\mathrm{a}}$ TNO Technical Sciences, Delft, The Netherlands; ${ }^{\mathrm{b}}$ Leiden Observatory, Leiden University, The \\ Netherlands.
}

\begin{abstract}
Over the last decade TNO has developed a deformable mirror concept using electromagnetic actuators with the main advantages of having very low non-linearity and hysteresis, low power consumption, and high inherent reliability of the actuators. TNO recently started a program to redesign the electromagnetic actuator to improve the actuator efficiency, allowing higher actuator force per volume and per wattage. The increased actuator efficiency gives improvement of the DM performance in terms of dynamical performance, actuation range, and power dissipation. With this technology various applications in the fields of ground-based astronomy and space missions are targeted.
\end{abstract}

Keywords: Adaptive Optics, Deformable Mirror, Actuators, Astronomy, Space,

\section{INTRODUCTION}

Modern astronomy often relies on adaptive optics to improve the overall imaging performance, utilizing a deformable mirror (DM) to compensate for optical wavefront aberrations stemming from for atmosphere turbulence. Besides ground based astronomy, Adaptive Optics is also emerging in other fields such as lithography, ophthalmology, and potentially for optical telescopes in future space missions.

Various DM systems are nowadays available, based on various actuation principles, such as electrostatic, piezoelectric, and electromagnetic [1]. Each actuation principle has its own advantages and disadvantages, and the best solution can change from application to application. Over the last decade TNO, in collaboration with the technical universities of Eindhoven and Delft, developed a deformable mirror concept using electromagnetic actuators [2,3]. Two prototype mirrors have been built with 61 and 427 actuators in a hexagonal grid with $6.3 \mathrm{~mm}$ pitch, as is shown in Figure 1. These prototypes have demonstrated the feasibility and potential of this electromagnetic actuated DM design.

The main assets of this technology are the large actuator strokes, low non-linearity and hysteresis, low power consumption, and the inherent high reliability of the actuators. The latter is due to the fact that the electromagnetic actuators do not suffer from wear, material aging or depolarization, which will be highly appreciated in high-end applications where uptime and operating cost are of high importance. Furthermore, various AO applications are emerging in which the stability and repeatability of the electromagnetic actuation principle can be highly beneficial. In many astronomy applications it is a strong wish, and sometimes a driving requirement, to be able to control the shape of the DM towards a predefined shape to allow imaging with reasonable quality in case the AO-loop is not active. Also Multi Object Adaptive Optics systems [3] and some forms of sensorless wavefront sensing [4] rely on open-loop shape control of the DM. This requires a highly stable and repeatable response of the DM which is one of the main assets of the electromagnetic actuation principle.

TNO recently started a program to redesign the electromagnetic actuator to improve the actuator efficiency, allowing for even higher actuator force per volume and per unit electrical power. The higher actuator efficiency allows further improvement of the DM performance in terms of stroke, dynamics, and power consumption. This paper discusses the progress on the electromagnetic DM development and gives an outlook on future development steps and potential applications.

*stefan.kuiper@tno.nl; phone +31 888664806; www.tno.nl 


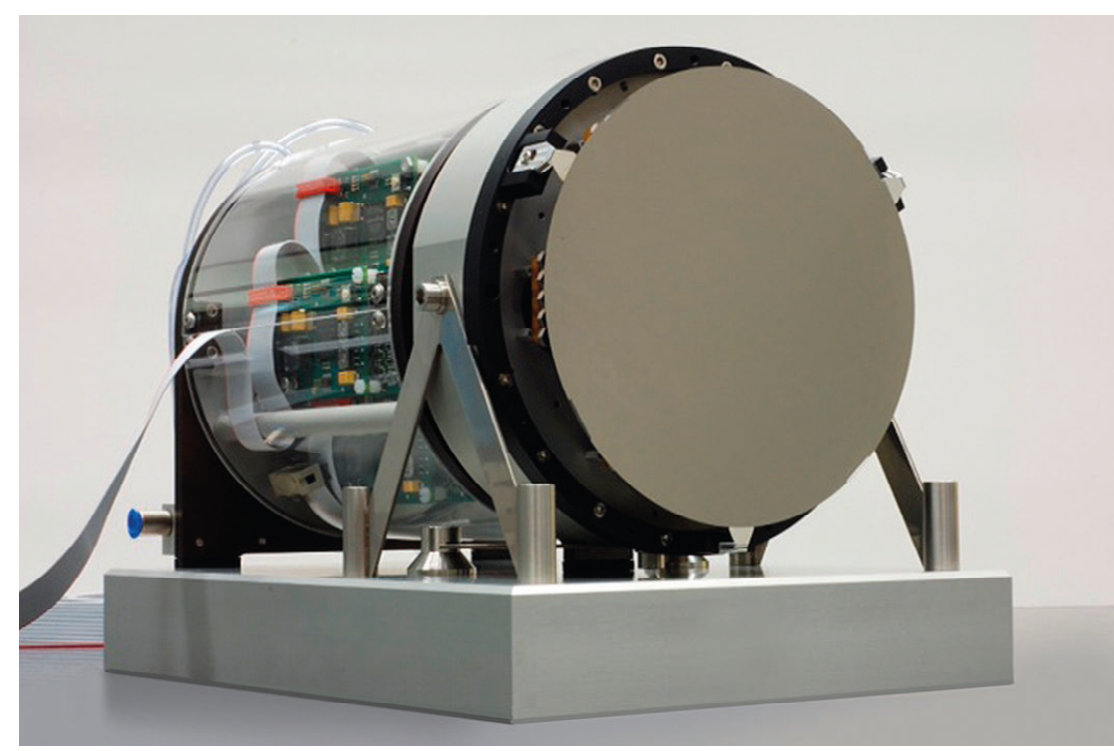

Figure 1: First deformable mirror prototype of TNO, containing 427 actuators $[2,3]$.

\section{DM TECHNOLOGY}

\subsection{Actuator design}

The heart of TNO's DM technology is formed by the renewed electromagnetic actuators, illustrated iin Figures 2 and 3. Figure 2 shows a miniaturized version of this actuator allowing a rectangular actuator grid of $4.3 \mathrm{~mm}$ pitch, while the Figure 3 shows a larger version of this actuator aimed at an actuator pitch of $18 \mathrm{~mm}$. These actuators are based on the principle of the variable reluctance motor, and further described in a patent [6]. The actuator consists of a soft magnetic iron core which is press-fitted into aluminum housing. A lever with the moving plunger is cut out via wire Electron Discharge Machining (EDM). The magnetic circuit is pre-energized via two permanent magnets, one above and one below the plunger. When the plunger is in the middle position, the forces from the two permanent magnets cancel each other. An electromagnetic coil generates an additional magnetic flux through the magnetic core which results in a net force on the plunger. The force on the plunger is transferred to the face sheet via a lever and a thin metal strut.

The actuator responses have been tested using a test-bench which mimics the face sheet stiffness via mechanical lever of which the displacement is measured via a capacitive sensor. The stiffness's of these levers are calibrated via a force probe. Figure 4 shows the measured force response of the $18 \mathrm{~mm}$ pitch actuator to a current of $\pm 150 \mathrm{~mA}$. As can be seen in Figure 3, the actuator shows an almost perfectly linear response up to the point that the magnetic core material starts to saturate. A linear region is selected between $\pm 150 \mathrm{mAmps}$, which corresponds to a force output of $\pm 8 \mathrm{~N}$ which is far sufficient to meet the required actuator strokes. The linearity over this range is measured to be within $99.5 \%$, and the hysteresis to be below $1 \%$. The high linearity and low hysteresis of the force response allow straightforward and highly repeatable control of the mirror shape. The motor constant is measured to be $52 \mathrm{~N} / \mathrm{A}$, which with a coil resistance of about $2.4 \Omega$ gives an actuator efficiency of $\alpha_{\text {act }}=38 \mathrm{~N} / \sqrt{W}$. This high actuator efficiency results in low power dissipation of the mirror as further discussed below.

Given the available actuation force range, the stiffness of the mechanical lever in the actuator can be tuned to find an optimal balance between range, mechanical resonance frequencies, power dissipation and mechanical coupling. A lower mechanical actuator stiffness results in a larger range and lower power dissipation, although the stiffness needs to be high enough to maintain sufficiently high actuator resonance and corresponding dynamical performance. Table 1 lists the DM performance based on a mechanical lever stiffness tuned to an actuator resonance of $2 \mathrm{kHz}$. Notice the relatively large actuator strokes, which are more than sufficient for most astronomy applications and provide a significant margin to prevent actuator clipping. 


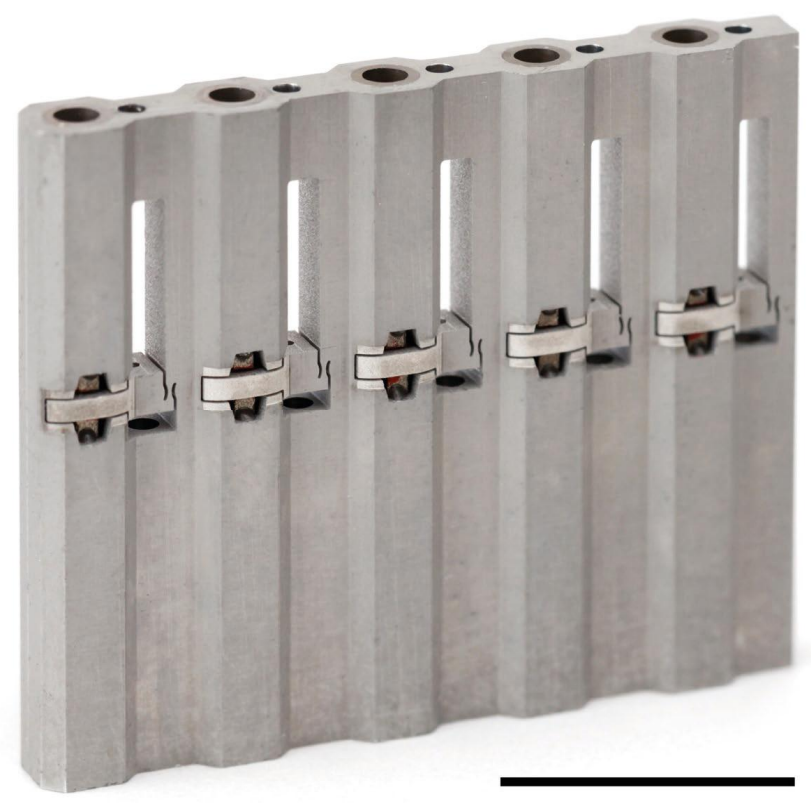

$10 \mathrm{~mm}$

Figure 2: A strip of 5 prototype electromagnetic actuators to be used in a deformable mirror. This strip enables a rectangular actuator grid with a pitch of $4.3 \mathrm{~mm}$.

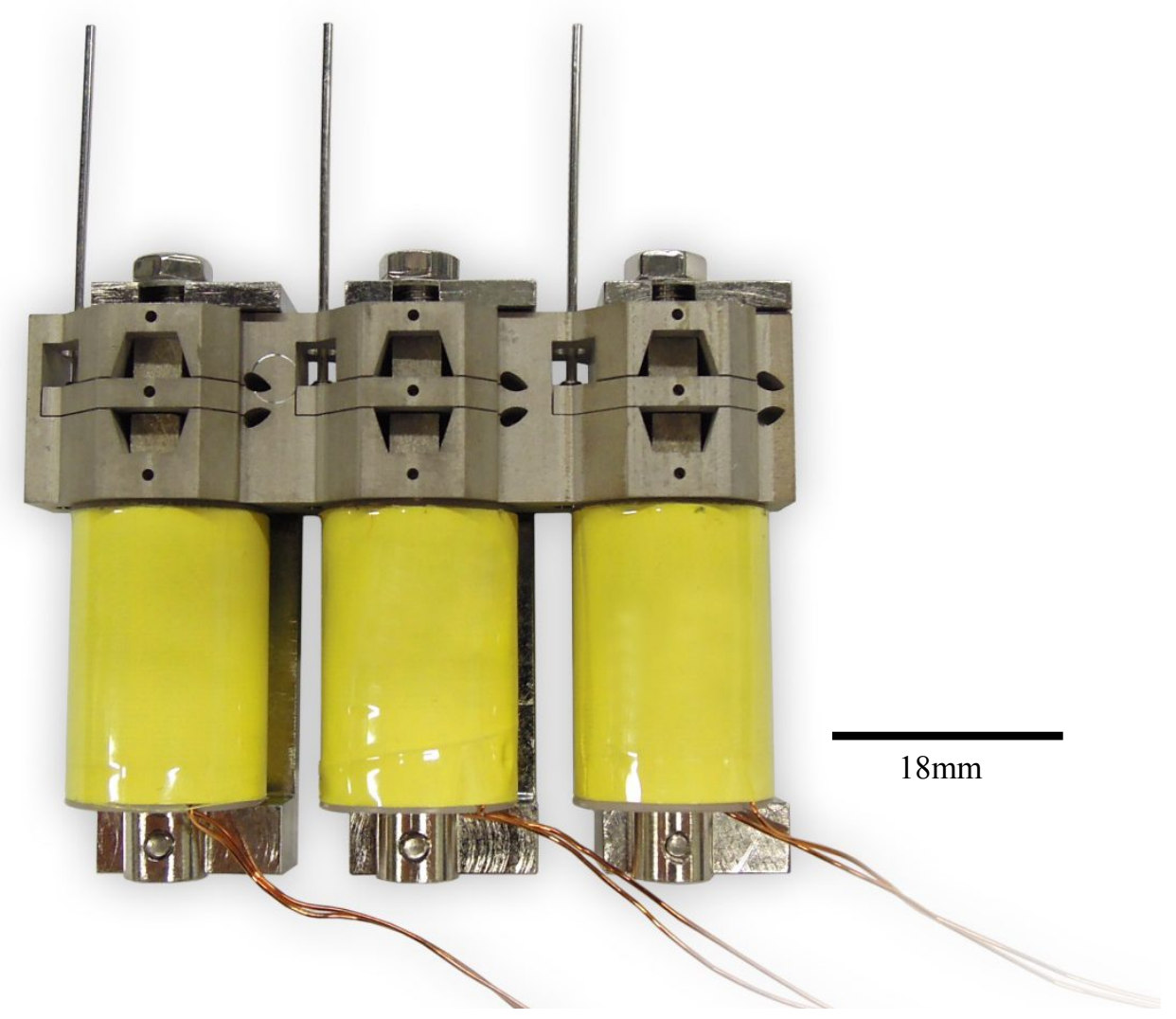

Figure 3: A strip of 3 prototype electromagnetic actuators to be used in a deformable mirror. This strip enables a rectangular actuator grid with a minimal pitch of $18 \mathrm{~mm}$. 


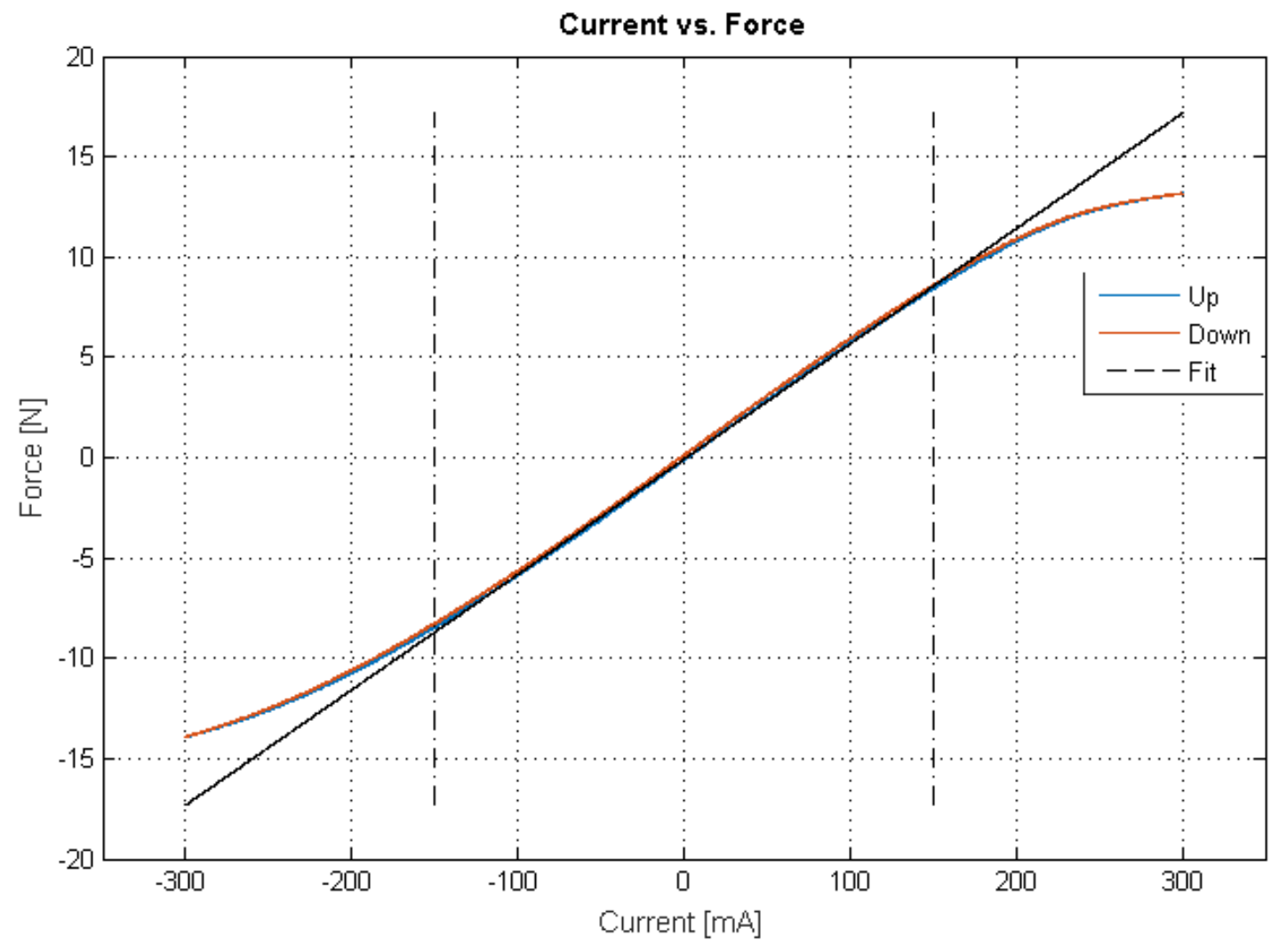

Figure 4: Actuator force response for a $\pm 300 \mathrm{~mA}$ driving current (blue and red curve). The black solid line shows the linear fit over the $\pm 150 \mathrm{~mA}$ range over which the force output is $\pm 8 \mathrm{~N}$. The linearity over this range is $99.5 \%$, and the hysteresis is less than $1 \%$.

Table 1: Specifications of a 200mm, 61 actuator DM based on the renewed electromagnetic actuator of Figure 3.

\begin{tabular}{|l|l|l|}
\hline Item & Value & Remarks \\
\hline Mirror diameter & $200 \mathrm{~mm}$ & Hexagonal grid \\
\hline Number of actuators & 61 & Hexagonal grid \\
\hline Pitch & $22 \mathrm{~mm}$ & At full actuator output. \\
\hline Inter actuator stroke & $12 \mu \mathrm{m}$ & Global piston movement of the DM \\
\hline Free actuator stroke & $46 \mu \mathrm{m}$ & $\begin{array}{l}\text { Movement of the neighboring actuators as a percentage } \\
\text { of the actuated actuator }\end{array}$ \\
\hline Actuator coupling & $24 \%$ & Vertical resonance of mirror on its support \\
\hline First mechanical resonance & $\sim 2 \mathrm{kHz}$ & Over full actuator force range \\
\hline Linearity & $99.5 \%$ & Over full actuator force range \\
\hline Hysteresis & $<1 \%$ & $\begin{array}{l}\text { Dissipation in actuator coils (apart from dissipation in } \\
\text { drive electronics) }\end{array}$ \\
\hline Maximum total Power dissipation & $3 \mathrm{Watts}$ & $\begin{array}{l}\text { Dissipation in actuator coils (apart from dissipation in } \\
\text { drive electronics) }\end{array}$ \\
\hline $\begin{array}{l}\text { Total power dissipation in normal } \\
\text { operation (over 57 actuators) }\end{array}$ & $\sim 0.3 \mathrm{Watts}$ & \multicolumn{2}{|l}{} \\
\hline
\end{tabular}




\subsection{DM assembly}

Figure 5 depicts an impression of a 200mm deformable mirror with 61 actuators. The actuators are manufactured in strips which stacked together form a rectangular or hexagonal actuator grid (see Figures 2 and 3). The actuators can also be manufactured as individual modules which are mounted separately on a support structure as shown in Figure 6 . The face sheet consists of a double sided polished glass membrane. The thickness of the face sheet is chosen to keep the quilting due to gravity below a couple of nanometers, while remaining sufficiently compliant to minimize the power consumption. The face sheet is connected to the actuators via mechanical struts, which provide high compliance in the actuation direction while being compliant in all other directions. The lateral movement of the face sheet is constrained with three folded leave springs orientated at 120 degrees intervals about the center of the mirror. This face sheet mounting results in minimal stresses and thus shape inaccuracy of the face sheet due to thermal elastic behavior or due to manufacturing tolerances.

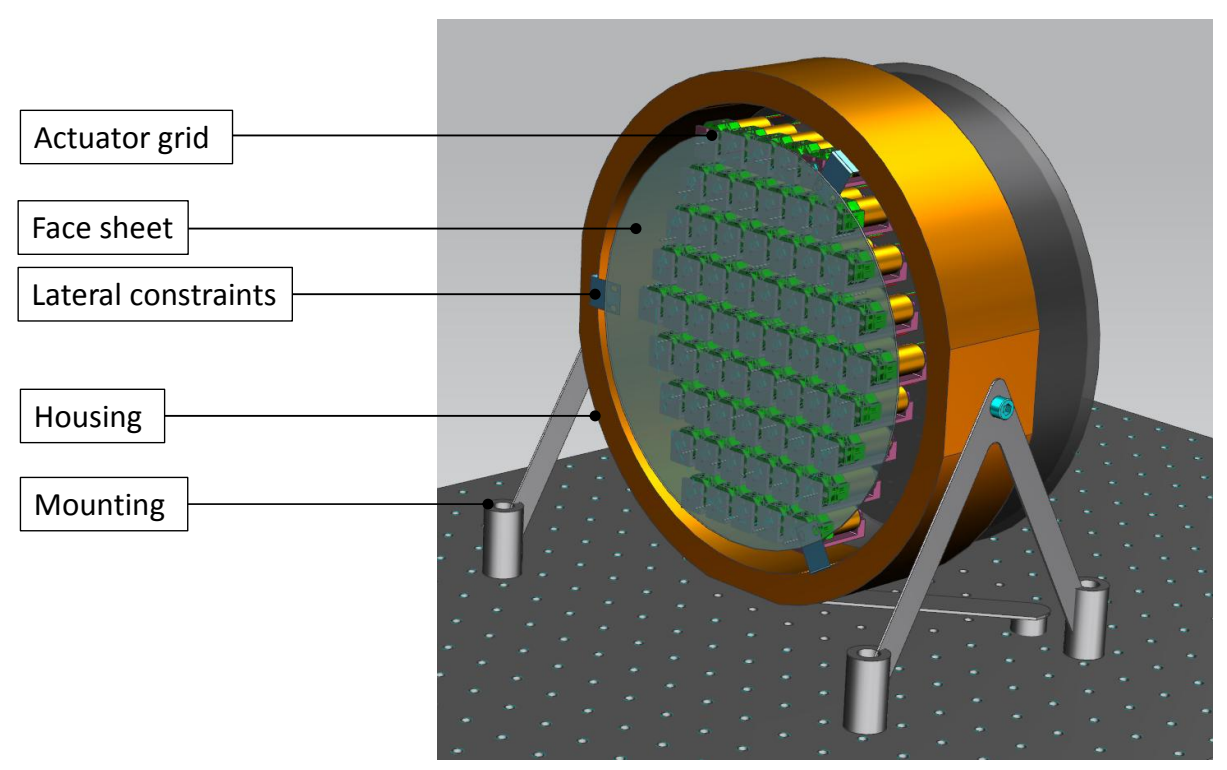

Figure 5: CAD impression of a DM based on the newly developed electromagnetic actuator. This DM contains 61 actuators in a $\sim 22 \mathrm{~mm}$ pitch hexagonal grid and a mirror diameter of $200 \mathrm{~mm}$.

\subsection{Reliability and serviceability}

One major advantage of the electromagnetic actuators is their inherent reliability, due to the lack of wear, aging or depolarization. This reliability is vital in high-end applications where uptime and operating cost are extremely important. On top of that, the electromagnetic actuators are relatively compliant, yielding high coupling towards the neighboring actuators. This has the advantage that even in the unlikely event of an actuator failure the neighboring actuators are still capable of compensating for the failed actuator to a large extent. This is in contrast to high stiffness actuators such as piezo actuators, where a broken actuator locks the face sheet around the location of the broken actuator.

In the design of the DM special attention has been given to the serviceability of the system to further reduce the operating cost and potential downtime during service intervals. The actuator coils are accessible from the backside of the mirror, and can be easily replaced if needed without impact on the rest of the system. Due to the removability of the actuator coils, the mechanical DM assembly can be put into a coating chamber without outgassing issues, which allows recoating of the mirror once the optical coating is degraded. The ability to recoat the face sheet can significantly reduce the operating cost, particularly in applications where unprotected coatings are preferred which typically degrade after periods of about two to three years. In case the full face sheet needs replacement, this can be done by separating the old face sheet via the strut interface, and bonding a new face sheet together with new struts on the actuators. Furthermore, the driving electronics are design modular, such that these can be easily replaced when needed without much impact on the uptime of the DM system. 


\section{DM DESIGN FOR SPACE APPLICATIONS}

Besides ground-based astronomy, TNO is also targeting space applications with the deformable mirror technology under development. The background for using adaptive optics in space applications is to compensate for wavefront aberrations stemming the light-weight, large telescope mirrors due to gravity release, thermal elastic effects, and initial manufacturing and alignment errors.

In the framework of an ESA TRP study, TNO is investigating the applicability of the electromagnetic DM technology for both space science and earth observation applications. Figure 6 shows an impression of a DM designed for space applications using 57 electromagnetic actuators. The dimensions used here are based on a $3.5 \mathrm{~m}$ aperture earth observation telescope, in which the DM is the $5^{\text {th }}$ mirror in the optical layout at a position conjugated to the M1 mirror. The mirror size at that location is an elliptical $610 \times 670 \mathrm{~mm}$ which requires an unusually large deformable mirror. TNO's electromagnetic DM concept allows for such large DM dimensions due to its high scalability. One important benefit of such large DM dimensions is the reduced anisoplanatic error, allowing for an effective wavefront corrections over larger fields of view with a single DM AO system.

The key asset of the designed electromagnetic actuators for space applications is their inherent reliability due to the lack of wear, aging or depolarization. On top of that, the drive electronics and actuator coils can be duplicated for redundancy without much impact on the overall systems complexity or mass. This redundancy on active components significantly increases the overall reliability and safety of the DM system, and is typically required for such active components in space applications.

Another important advantage of this DM technology for space applications is the low power dissipation, which is due to the high actuator efficiency, and the fact that the inductive load of the coils can be driven by highly efficient Pulse Width Modulated Amplifiers. The total power dissipation for the 57 actuator DM depicted in Figure 6 is estimated around 5 Watts of which about 0.3 Watts is dissipated in the actuator. Such low power dissipation fits well within the requirements for space applications.

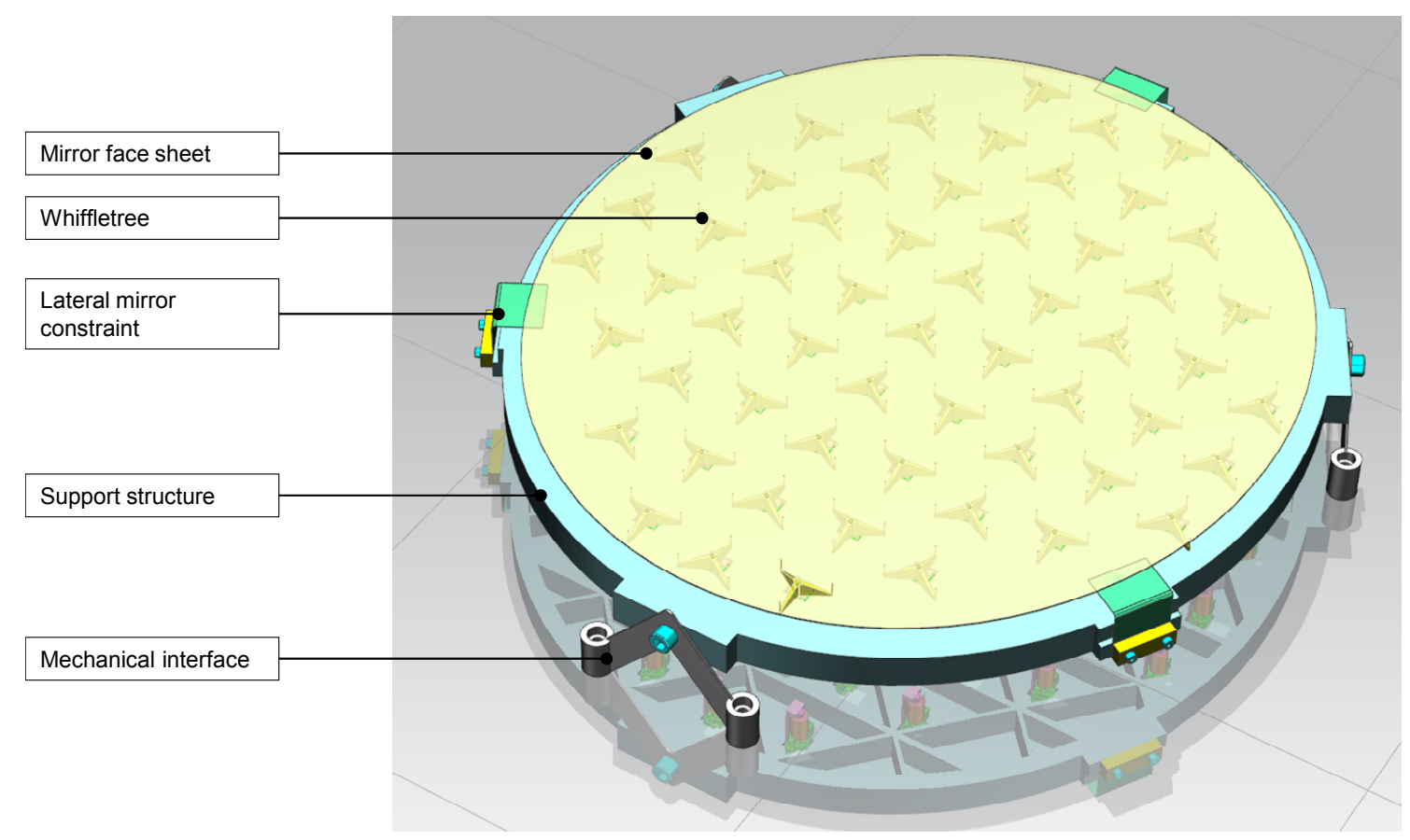

Figure 6: Impression of the DM for space applications based on 57 electromagnetic actuators as shown in Figure 3. The mirror dimensions are an elliptical 610x670mm. 


\section{CONCLUSIONS}

TNO is developing a deformable mirror technology based on a renewed electromagnetic actuation principle. The key benefits of this technology are high reliability, highly linear and repeatable actuator response, and low power dissipation. The DM concept is scalable from an actuator pitch of around $4 \mathrm{~mm}$ up to more than $70 \mathrm{~mm}$. With this technology TNO is aiming at both ground based and space optical telescope applications.

After testing of the renewed actuator and several face sheet integration tests, the next steps in this development are the building and testing a full prototype DM based on this renewed actuator technology.

\section{Acknowledgements}

TNO wishes to thank Roger Hamelinck and Christian Werner of Entechna Engineering for their valuable contributions in the design of the renewed actuator technology, Xander Janssen and Mathieu Breukers of VDL ETG for their contributions on the design for assembly and integration of the actuator structures, and Alex van den Heuvel of Technolution for his inputs on the design and implementation on the driving electronics.

\section{References}

[1] Madec, P.-Y. "Overview of deformable mirror technologies for adaptive optics and astronomy.” Proc. SPIE 84470, 844705-844705-18 (2012)

[2] Hamelinck, R.F.M.M. ; Ellenbroek, R; Rosielle, N; Steinbuch, M; Verhaegen, M, et al. "Validation of a new adaptive deformable mirror concept", Proc. SPIE 7015, 70150Q (2008)

[3] Hamelinck, R.F.M.M. [Adaptive deformable mirror], Diss. Technical University of Eindhoven (2010)

[4] Guzman, C. [Technologies for Astronomical Wide-Field Adaptive Optics], Diss. Durham University (2010)

[5] Booth, M. J. "Wavefront sensorless adaptive optics for large aberrations." Optics letters, 32.1, 5-7 (2007)

[6] Hamelinck, R.F.M.M.; Werner, C. [Reluctance transducer, WO 2014042525] (2014) 